\title{
A new classification of the Arctic spring transition in the middle atmosphere
}

V. Matthias, G.Stober, A.Kozlovsky, M.Lester, E. Belova and J. Kero

Stratosphere
- stratospheric final warming (SFW)
- SFW onset days vary by about 2 month
- common classification: early/late or $10 \mathrm{hPa} / 1 \mathrm{hPa}$-first
\[ \text { Mesosphere } \]
- final wind reversal earlier and less variable as in
stratosphere, mostly propagates downward
- studies are much rarer, less systematic and decoupled
from stratospheric investigations

\section{What is missing?}

- systematic studies of the spring transition covering the whole middle atmosphere - common classification do not consider all SFWs - it is not clear if and how pre-winter conditions have an impact on when and how spring transition take place

\section{What we want to}

- introduce a new type of classification based on temporal-vertical evolution of polar vortex

- includes: stratosphere and mesosphere, all spring transitions, SSWs in preceding winter timing of major SSW in preceding winter

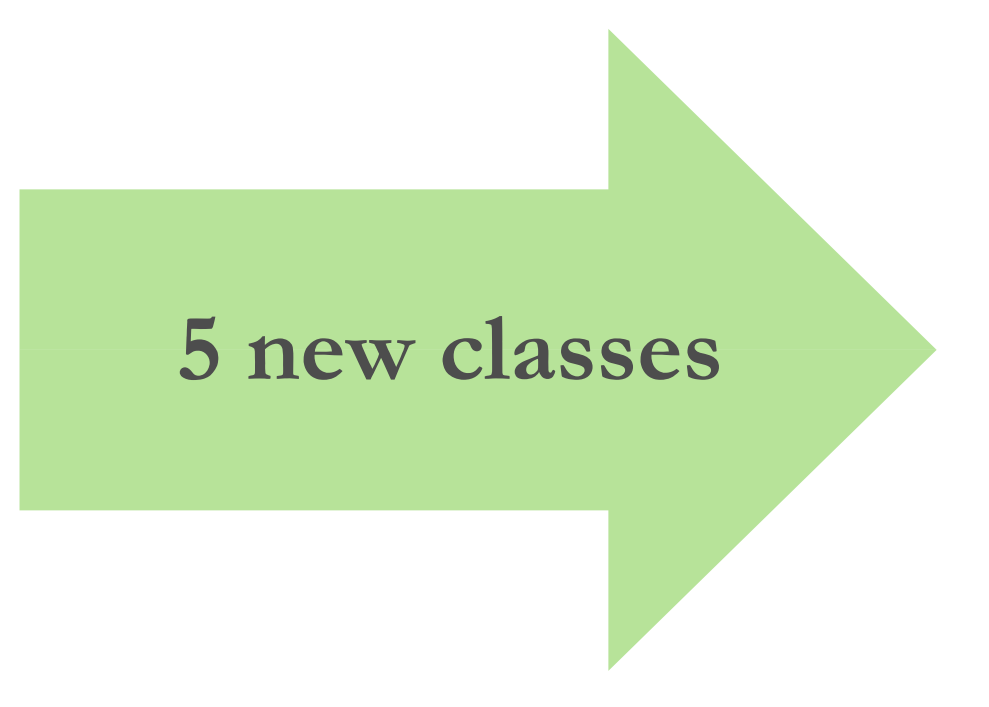

downward propagating negative NAM
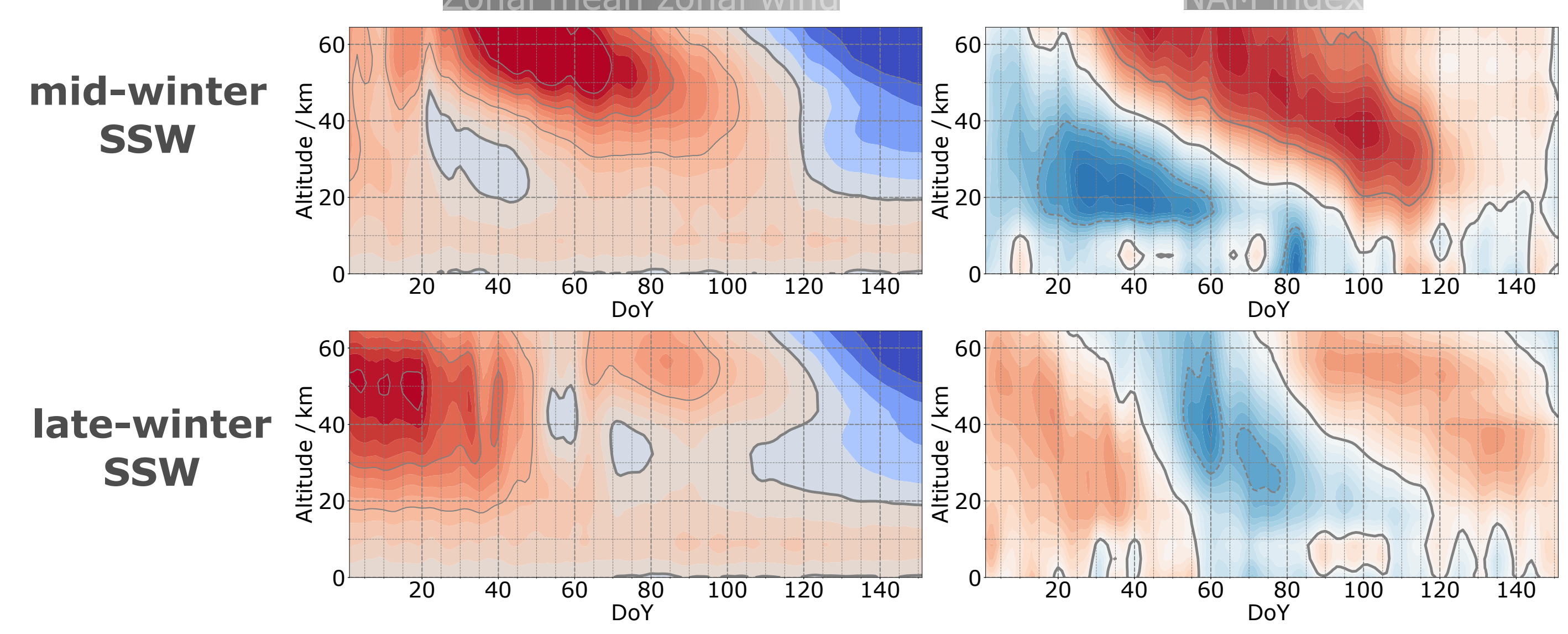

early-spring $\xi$ ssw
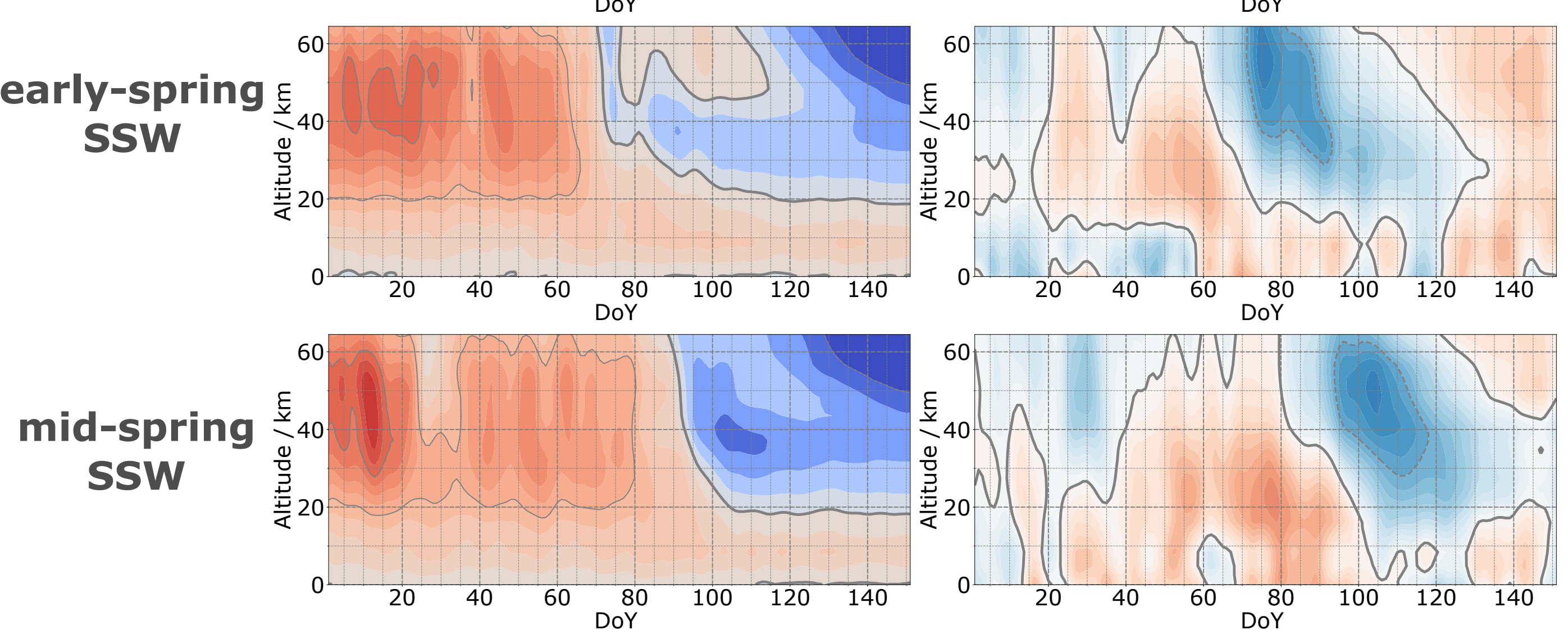

no negative $\underset{\text { NAM }}{\stackrel{\frac{E}{4}}{\frac{E}{2}} 40}$
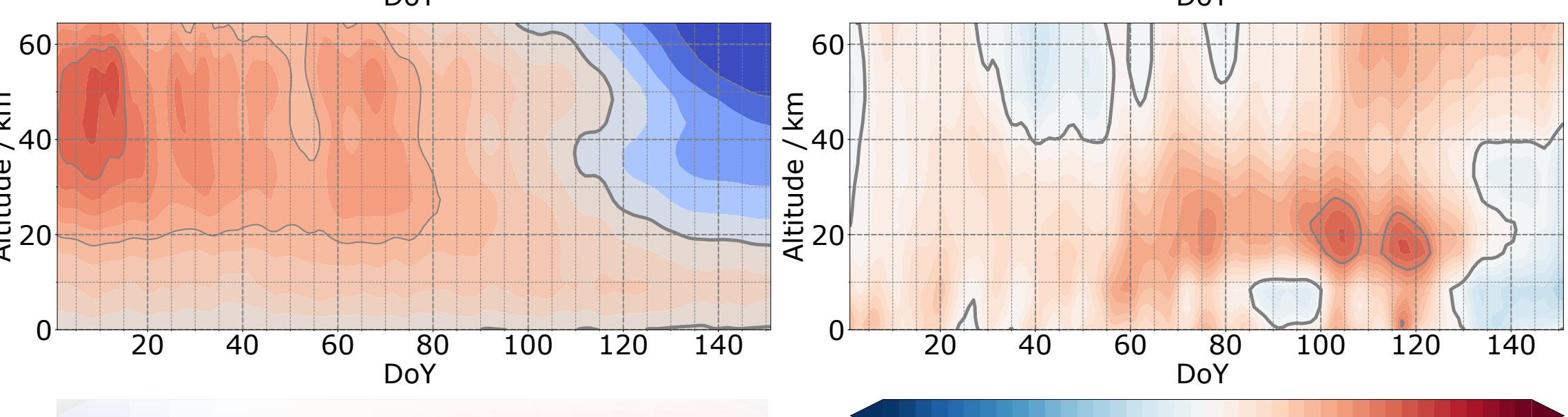